Pacific Journal of Mathematics

ON A COMMUTATOR RESULT OF TAUSSKY AND 


\title{
ON A COMMUTATOR RESULT OF \\ TAUSSKY AND ZASSENHAUS
}

\author{
MARVin MARCUS ${ }^{1}$ AND N. A. KHAN ${ }^{2}$
}

1. Introduction and results. Let $M_{n}$ denote the set of $n$-square matrices over a field $F$. For $A, B$ in $M_{n}$ let $[A, B]=A B-B A^{\prime}$, where $A^{\prime}$ is the transpose of $A$ and define inductively

$$
[A, B]_{k}=\left[A,[A, B]_{k-1}\right] \text {. }
$$

If $P^{-1} J P=A$, then

$$
[A, X]=\left[P^{-1} J P, X\right]=P^{-1}\left[J, P X P^{\prime}\right]\left(P^{-1}\right)^{\prime},
$$

and similarly

$$
[A, X]_{k}=P^{-1}\left[J, P X P^{\prime}\right]_{k}\left(P^{-1}\right)^{\prime} .
$$

Now for a fixed $A$ let $T$ be the linear map of $M_{n}$ into itself defined by

$$
T(Y)=[A, Y]
$$

and (1.1) implies that

$$
T^{k}(Y)=[A, Y]_{k} .
$$

In a recent paper [1], Taussky and Zassenhaus showed that $A$ is nonderogatory if and only if any nonsingular $X$ in the null space of $T$ is symmetric. In this note we investigate the structure of the null space of both $T$ and $T^{2}$ for arbitrary $A$.

Enlarge the field $F$ to include $\lambda_{i}, i=1, \cdots, p$, the distinct eigenvalues of $A$, and let $\left(x-\lambda_{i}\right)^{e_{i j}}, j=1, \cdots, n_{i}, e_{i 1}>\cdots>e_{i n_{i}}, i=1, \cdots, p$ be the distinct elementary divisors of $A$ where $\left(x-\lambda_{i}\right)^{e_{i j}}$ appears with multiplicity $r_{i j}$. Set $m_{i}=\sum_{j=1}^{n_{i}} r_{i j} e_{i j}$, the algebraic multiplicity of $\lambda_{i}$. Let $\eta(T)$ denote the null space of $T, \sigma(T)$ denote the subspace of symmetric matrices in $\eta(T)$, and $\gamma(T)$ denote the subspace of skew-symmetric matrices in $\eta(T)$. We show that

$$
\begin{gathered}
\operatorname{dim} \eta(T)=\sum_{i=1}^{p}\left[\sum_{j=1}^{n_{i}}\left(r_{i j}^{2} e_{i j}+2 r_{i j} \sum_{k=j+1}^{n_{i}} r_{i k} e_{i k}\right)\right], \\
\operatorname{dim} \sigma(T)=\frac{1}{2} \sum_{i=1}^{p}\left[\sum_{j=1}^{n_{i}}\left\{r_{i j}\left(r_{i j}+1\right) e_{i j}+2 r_{i j} \sum_{k=j+1}^{n_{i}} r_{i k} e_{i k}\right\}\right],
\end{gathered}
$$

Received December 17, 1959. The work of this author was supported by U. S. National Science Foundation Grant, NSF-G5416. The second author is a Postdoctorate Fellow of the National Research Council of Canada. The authors are grateful to Professor O. Taussky for her helpful suggestions. 


$$
\operatorname{dim} \eta\left(T^{2}\right)=\sum_{i=1}^{p}\left[\sum_{j=1}^{n_{i}}\left\{r_{i j}^{2}\left(2 e_{i j}-1\right)+4 r_{i j} \sum_{k=j+1}^{n_{i}} r_{i k} e_{i k}\right\}\right]
$$

$$
\operatorname{dim} \sigma\left(T^{2}\right)=\frac{1}{2} \sum_{i=1}^{p}\left[\sum_{j=1}^{n_{i}}\left\{r_{i j}^{2}\left(2 e_{i j}-1\right)+r_{i j}+4 r_{i j} \sum_{k=j+1}^{n_{i}} r_{i k} e_{i k}\right\}\right] \text {. }
$$

In case $A$ is nonderogatory, $n_{i}=1, r_{i j}=1, i=1, \cdots, p$ and (1.4) and (1.5) reduce to

$$
\operatorname{dim} \eta(T)=n=\operatorname{dim} \sigma(T) .
$$

Thus every matrix $X$ satisfying

$$
A X=X A^{\prime}
$$

where $A$ is non-derogatory is symmetric, the result in [1]. Moreover, if every matrix $X$ satisfying (1.8) is symmetric then $\operatorname{dim} \eta(T)=\operatorname{dim} \sigma(T)$. Using the formulas (1.4) and (1.5) we see that this condition implies that

$$
\sum_{i=1}^{p} \sum_{j=1}^{n_{i}}\left(r_{i j}^{2}-r_{i j}\right) e_{i j}+2 \sum_{i=1}^{p} r_{i j} \sum_{k=j+1}^{n_{i}} r_{i k} e_{i k}=0 .
$$

Now since $r_{i j}, e_{i j}$ and $n_{i}$ are all positive integers we conclude that $r_{i j}=1, j=1, \cdots, n_{i}$ and $n_{i}=1$. That is, there is only one elementary divisor corresponding to each eigenvalue. Hence, if every matrix $X$ satisfying (1.8) is symmetric then $A$ is non-derogatory, a result also found in [1].

We also show in this case that $\eta(T)$ consists of matrices of the form $P X P^{\prime}$ where $P$ is fixed (depending on $A$ ) and $X$ is persymmetric, (i.e. all the entries of $X$ on each line perpendicular to the main diagonal are equal).

We next note that $\eta(T)=\sigma(T)+\gamma(T)$ (direct) and $\eta\left(T^{2}\right)=\sigma\left(T^{2}\right)+$ $\gamma\left(T^{2}\right)$ (direct). The first statement is easy to show; we indicate the brief proof of the second statement:

Since $X=\frac{X+X^{\prime}}{2}+\frac{X-X^{\prime}}{2}$, if $X \in \eta\left(T^{2}\right)$, then

$$
\begin{aligned}
T^{2}\left(X+X^{\prime}\right) & =\left[A,\left[A, X+X^{\prime}\right]\right] \\
& =\left[A,[A, X]+\left[A, X^{\prime}\right]\right] \\
& =[A,[A, X]]+\left[A,\left[A, X^{\prime}\right]\right] \\
& =T^{2}(X)-\left[A,[A, X]^{\prime}\right] \\
& =[A,[A, X]]^{\prime} \\
& =\left(T^{2}(X)\right)^{\prime}=0 .
\end{aligned}
$$

Similarly, $T^{2}\left(X-X^{\prime}\right)=0$. Thus any $X \in \eta\left(T^{2}\right)$ is expressible uniquely as a sum of two elements, one in $\sigma\left(T^{2}\right)$ and the other in $\gamma\left(T^{2}\right)$. Hence 


$$
\begin{aligned}
& \operatorname{dim} \gamma(T)=\operatorname{dim} \eta(T)-\operatorname{dim} \sigma(T) \\
= & \frac{1}{2} \sum_{i=1}^{p}\left[\sum_{j=1}^{n_{i}}\left\{r_{i j}\left(r_{i j}-1\right) e_{i j}+2 r_{i j} \sum_{k=j+1}^{n_{i}} r_{i k} e_{i k}\right\}\right], \\
& \operatorname{dim} \gamma\left(T^{2}\right)=\operatorname{dim} \eta\left(T^{2}\right)-\operatorname{dim} \sigma\left(T^{2}\right) \\
= & \frac{1}{2} \sum_{i=1}^{p}\left[\sum_{j=1}^{n_{i}}\left\{r_{i j}^{2}\left(2 e_{i j}-1\right)-r_{i j}+4 r_{i j} \sum_{k=j+1}^{n_{i}} r_{i k} e_{i k}\right\}\right] .
\end{aligned}
$$

In case $A$ is non-derogatory, (1.6), (1.7) and (1.10) reduce to

$$
\begin{aligned}
& \operatorname{dim} \eta\left(T^{2}\right)=2 n-p, \\
& \operatorname{dim} \sigma\left(T^{2}\right)=n, \\
& \operatorname{dim} \gamma\left(T^{2}\right)=n-p .
\end{aligned}
$$

We thus conclude that unless all the eigenvalues of $A$ are distinct $(p=n)$ there exist skew-symmetric matrices $X$ satisfying

$$
A^{2} X-2 A X A^{\prime}+X\left(A^{\prime}\right)^{2}=0 .
$$

If $p=n$, and $A$ is non-derogatory

$$
\operatorname{dim} \eta\left(T^{2}\right)=n=\operatorname{dim} \sigma\left(T^{2}\right)
$$

and any matrix $X$ satisfying (1.11) is symmetric.

On the other hand suppose

$$
\operatorname{dim} \eta\left(T^{2}\right)=\operatorname{dim} \sigma\left(T^{2}\right) .
$$

From (1.6) and (1.7) we conclude that

$$
\sum_{i=1}^{p}\left[\sum_{j=1}^{n_{i}}\left\{r_{i j}^{2}\left(2 e_{i j}-1\right)-r_{i j}+4 r_{i j} \sum_{k=j+1}^{n_{i}} r_{i k} e_{i k}\right\}\right]=0 .
$$

Hence $n_{i}=1, r_{i j}=1, e_{i k}=1$ and we conclude that $p=n$. That is, if every matrix $X$ satisfying (1.11) is symmetric then the eigenvalues of $A$ are distinct.

We show finally (Theorem 2) that if $A$ is an $n$-square matrix with $p$ distinct eigenvalues then both $\operatorname{dim} \gamma(T)$ and $\operatorname{dim} \gamma\left(T^{2}\right)$ are at most $\frac{1}{2}(n-p)(n-p+1)$. Moreover, for each $p$ this bound is best possible.

Thus if there exists a skew-symmetric solution of (1.8) or (1.11), then $A$ has multiple eigenvalues, without the assumption that $A$ is nonderogatory.

II. Proofs. Let $E_{i j} \in M_{n}$ be the matrix with 1 in position $i, j$ and 0 elsewhere. With respect to this basis, ordered lexicographically, it may be checked that $T$ has the matrix representaion 


$$
T=I \otimes A-A \otimes I
$$

where $\otimes$ indicates Kronecker product.

From (1.2) we may take $A$ to be in Jordan canonical form $J$, since $[A, X]_{k}=0$ if and only if $\left[J, P X P^{\prime}\right]_{k}=0$ and $P X P^{\prime}$ is symmetric if and only if $X$ is. We write

$$
J=\sum_{s=1}^{p} \cdot J_{s}
$$

where

$$
J_{s}=\lambda_{s} I_{m_{s}}+\sum_{t=1}^{n_{s}} \cdot \sum_{1}^{r_{s t}} \cdot U_{e_{s t}}
$$

$\Sigma^{*}$ indicates direct sum, $I_{t}$ is a $t$-square identity matrix, $U_{t}$ is $t$-square auxiliary unit matrix (i.e. 1 in the superdiagonal and 0 elsewhere) and ${ }^{r_{j i}} \cdot U_{e_{s t}}$ is the direct sum of $U_{e_{s t}}$ with itself $r_{i j}$ times.

By a routine computation we see that

$$
T^{k}(Y)=0
$$

if and only if

$$
\sum_{\alpha=0}^{k}\left(\begin{array}{l}
k \\
\alpha
\end{array}\right)(-1)^{\alpha} J_{s}^{k-\alpha} Y_{s t}\left(J_{t}^{\prime}\right)^{\alpha}=0, \quad s, t=1, \cdots, p,
$$

where $Y=\left(Y_{s t}\right), s, t=1, \cdots, p$ is a partitioning of $Y$ conformal with the partitioning of $J$ given by (2.2).

For $s \neq t$, it is clear that the matrix representation of (2.4),

$$
\left(I_{m_{t}} \otimes J_{s}-J_{t} \otimes I_{m_{s}}\right)^{k}
$$

has the single nonzero eigenvalue $\left(\lambda_{s}-\lambda_{t}\right)^{k}$ and thus $Y_{s t}=0$. Hence we need only consider the equation (2.4) for $s=t$. We may again partition $Y_{s s}$ conformally with $J_{s}$ in (2.3). We are thus led to consider the null space of the mapping

$$
\left(I_{e_{s i}} \otimes U_{e_{s j}}-U_{e_{s i}} \otimes I_{e_{s j}}\right)^{k} .
$$

Lemma 1. Let $T=I_{m} \otimes U_{n}-U_{m} \otimes I_{n}$. Then

$$
\begin{gathered}
\operatorname{dim} \eta(T)=\min (m, n), \\
\operatorname{dim} \eta\left(T^{2}\right)= \begin{cases}2 \min (m, n), & \text { if } m \neq n \\
2 n-1, & \text { if } m=n .\end{cases}
\end{gathered}
$$

Proof. Suppose $n \leqq m$ and that $T(X)=0$. Let $x_{1}, \cdots, x_{m}$ be the column $n$-vectors of $X$. Then we have 


$$
\begin{aligned}
U_{n} x_{j}-x_{j+1} & =0, \quad j=1,2, \cdots, m-1, \\
U_{n} x_{m} & =0 .
\end{aligned}
$$

For $r=1,2, \cdots, n-1$ consider the $(r-j+1)$ coordinate of $(2.8)$ for $j=1, \cdots, r$ and we conclude that

$$
x_{r+1,1}=x_{r, 2}=\cdots=x_{1, r+1}=c_{r+1} .
$$

Next consider the $(n-j+1)$ coordinate of $(2.8)$ for $j=1, \cdots, n$ to obtain

$$
0=x_{n 2}=x_{n-1,3}=\cdots=x_{1, n+1} .
$$

Similarly we see that the remaining elements of $X$ are zero. Hence we find that the $j$ th column of the $n \times m$ matrix $X$ is the transpose of the $n$-vector

$$
\left[c_{j}, c_{j+1}, \cdots, c_{n}, 0, \cdots, 0\right]
$$

for $j=1,2, \cdots, n$. The other $m-n$ columns are zero.

In case $n \geqq m$, it is easy to check that the $j$ th row of $X$ is the $m$-vector

$$
\left[c_{j}, c_{j+1}, \cdots, c_{m}, 0, \cdots, 0\right]
$$

for $j=1,2, \cdots, m$. The other $n-m$ rows are zero.

This establishes (2.6). To prove (2.7) let $T^{2}(X)=0$ and $x_{1}, x_{2}, \cdots, x_{m}$ be the column $n$-vectors of $X$. Let us consider the following cases:

(i) $m=n$.

We have

$$
U_{n}^{2} x_{n}=0, U_{n}^{2} x_{n-1}=2 U_{n} x_{n}
$$

and

$$
U_{n}^{2} x_{j}-2 U_{n} x_{j+1}+x_{j+2}=0, j=1,2, \cdots, n-2 .
$$

Solving these equations recursively we find that the lst, 2nd and $j$ th rows of $X$ are respectively

$$
\begin{gathered}
{\left[x_{11}, x_{12}, \cdots, x_{1, n-2}, x_{1, n-1}, x_{1 n}\right],} \\
{\left[x_{21}, x_{22}, \cdots, x_{2, n-2}, x_{2, n-1}, 0\right]}
\end{gathered}
$$

and

$$
\begin{array}{r}
(j-1)\left[x_{2, j-1}, x_{2, j}, \cdots, x_{2, n-1}, 0, \cdots, 0\right] \\
-(j-2)\left[x_{1, j}, x_{1, j+1}, \cdots, x_{1, n}, 0, \cdots, 0\right],
\end{array}
$$

for $j=3,4, \cdots, n$.

The number of arbitrary parameters in $X$ is $2 n-1$. 
(ii) $n<m$.

Here we have the following equations:

$$
\begin{gathered}
U_{n}^{2} x_{j}-2 U_{n} x_{j+1}+x_{j+2}=0, j=1,2, \cdots, m-2 \\
U_{n}^{2} x_{m-1}-2 U_{n} x_{m}=0 \\
U_{n}^{2} x_{m}=0
\end{gathered}
$$

and by solving recursively again we find that the 1 st, 2 nd and $j$ th rows of $X$ are respectively the $m$-vectors

$$
\begin{gathered}
{\left[x_{11}, \cdots, x_{1, n-1}, x_{1, n}, n x_{n, 2}, 0, \cdots, 0\right],} \\
{\left[x_{21}, \cdots, x_{2, n-1},(n-1) x_{n, 2}, 0,0, \cdots, 0\right]}
\end{gathered}
$$

and

$$
\begin{aligned}
& {\left[(j-1) x_{2, j-1}, \cdots,(j-1) x_{2, n-1},(n-j+1) x_{n, 2}, 0, \cdots, 0\right]} \\
& \quad-(j-2)\left[x_{1, j}, \cdots, x_{1, n}, 0,0, \cdots, 0\right]
\end{aligned}
$$

for $j=3,4, \cdots, n$.

In case $n>m$, by similar computation we find that the 1st, 2nd and $j$ th rows of $X$ are respectively

$$
\begin{gathered}
{\left[x_{11}, \cdots, x_{1, m-2}, x_{1, m-1}, x_{1 m}\right],} \\
{\left[x_{21}, \cdots, x_{2, m-2}, x_{2, m-1}, x_{2 m}\right]}
\end{gathered}
$$

and

$$
\begin{array}{r}
(j-1)\left[x_{2, j-1}, \cdots, x_{2, m-1}, x_{2 m}, 0, \cdots, 0\right] \\
-(j-2)\left[x_{1, j}, \cdots, x_{1, m}, 0,0, \cdots, 0\right]
\end{array}
$$

for $j=3,4, \cdots, m+1$. The remaining $n-m-1$ rows are zero.

From case (ii), we observe that the number of parameters in $X$ is $2 \min (m, n)$.

We now state and prove the following

Lemma 2. Let $A$ be an n-square matrix with the single eigenvalue $\lambda$ and let $(x-\lambda)^{n_{i}}$ be an elementary divisor of $A$ of multiplicity $r_{i}$, $i=1, \cdots, p, n_{1}>\cdots>n_{p}$. Then the most general matrix $X$ satisfying (1.11) has

$$
\sum_{i=1}^{p}\left[r_{i}^{2}\left(2 n_{i}-1\right)+4 r_{i} \sum_{j=i+1}^{p} r_{j} e_{j}\right]
$$

arbitrary parameters.

Moreover if $X$ is symmetric it contains 


$$
\frac{1}{2} \sum_{i=1}^{p}\left[r_{i}^{2}\left(2 n_{i}-1\right)+r_{i}+4 r_{i} \sum_{j=i+1}^{p} r_{j} n_{j}\right]
$$

parameters.

Proof. Without any loss of generality we can assume that

$$
A=\sum_{i=1}^{p} \cdot \sum_{j=1}^{r_{i}} \cdot U_{i}
$$

where $\Sigma \cdot U_{i}$ indicates the direct sum of $U_{i}$ with itself $r_{i}$ times. We partition $X$ conformally with $A$ in (2.12) and observe that the equation

$$
U_{i}^{2} X_{i j}-2 U_{i} X_{i j} U_{j}^{\prime}+X_{i j}\left(U_{j}^{\prime}\right)^{2}=0
$$

determines the structure of any block $X_{i j}$ in the partitioning of $X$.

From case (i) of Lemma 1 , we conclude that any block $X_{i j}$ corresponding to equal $U_{i}$ 's contains $2 n_{i}-1$ arbitrary parameters and there are $r_{i}^{2}$ such blocks. Also from case (ii) any block in $X$ that corresponds to $U_{i}$ and $U_{j}, i<j$, contains $2 n_{j}$ arbitrary parameters. Hence the total number of parameters in $X$ is given by (2.10).

In order to find the number of parameters in a symmetric $X$ we first consider a diagonal block. Its structure has been discussed in Lemma 1 , case (i). We observe that if this matrix is symmetric, the number of parameters in it reduces from $2 n_{i}-1$ to $n_{i}$.

Then we consider two symmetrically placed off-diagonal blocks $X_{i j}$ and $X_{j i}$ of orders $n_{i} \times n_{j}$ and $n_{j} \times n_{i}$ respectively. If $X$ is to be symmetric then by equating the terms of $X_{i j}$ and $X_{j i}$ which are symmetrically placed about the main diagonal of $X$, the number of arbitrary parameters in $X_{i j}$ and $X_{j i}$ reduces from $2\left(2 n_{j}\right)$ to $2 n_{j}$. If $X_{i j}$ and $X_{j i}$ are of order $n_{i} \times n_{i}$ then the number of parameters reduces from $2\left(2 n_{i}-1\right)$ to $2 n_{i}-1$.

We are now in a position to sum the number of parameters in $X$ if it is symmetric and satisfies (1.11). There are $r_{i}$ blocks in the main diagonal, each of order $n_{i}, i=1, \cdots, p$. The number of parameters in each of these blocks is $n_{i}$. There are $r_{i}\left(r_{i}-1\right) / 2$ other square blocks of order $n_{i}$. Each of them contains $\left(2 n_{i}-1\right)$ parameters. Thus

$$
\frac{1}{2} \sum_{i=1}^{p}\left\{r_{i}^{2}\left(2 n_{i}-1\right)+r_{i}\right\}
$$

is the number of parameters in all those blocks of $X$ which are square. Since any block of order $n_{i} \times n_{j}$ where $n_{i}>n_{j}$ contains $2 n_{j}$ parameters, and since we are considering $X$ to be symmetric, we conclude that the total number of arbitrary parameters in $X$ is given by (2.11).

We can similarly prove the following

Lemma 3. Let $A$ be the matrix given in Lemma 2. Then the most 
general matrix $X$ satisfying (1.8) has

$$
\sum_{i=1}^{p}\left(r_{i}^{2} n_{i}+2 r_{i} \sum_{j=i+1}^{p} r_{j} n_{j}\right)
$$

arbitrary parameters.

Moreover if $X$ is symmetric, it contains

$$
\frac{1}{2} \sum_{i=1}^{p}\left[r_{i}\left(r_{i}+1\right) n_{i}+2 r_{i} \sum_{j=i+1}^{p} r_{j} n_{j}\right]
$$

parameters.

We now state and prove the following

THEOREM 1. Let $A$ be an n-square matrix with distinct eigenvalues $\lambda_{1}, \cdots, \lambda_{p}$ and let $\left(x-\lambda_{i}\right)^{e_{i j}}, j=1, \cdots, n_{i}, e_{i 1}>\cdots>e_{i n_{i}}$ be the elementary divisors of $A$ corresponding to $\lambda_{i}$, where each $\left(x-\lambda_{i}\right)^{e_{i j}}$ has been repeated $r_{i j}$ times. Then (1.4), (1.5), (1.6) and (1.7) hold.

Proof. It was pointed out earlier that if $Y=\left(Y_{r s}\right), r, s=1, \cdots, p$ is the partitioning of $Y$ conformal with the partitioning of $J$ in (2.2), then all the off-diagonal blocks are zero. Hence we have simply to find the number of parameters in $Y_{i i}, i=1, \cdots, p$.

As proved in Lemma 2, the number of parameters in $Y_{i i}$ is

$$
\sum_{j=1}^{n_{i}}\left[r_{i j}^{2}\left(2 e_{i j}-1\right)+4 r_{i j} \sum_{k=j+1}^{n_{i}} r_{i k} e_{i k}\right] \text {. }
$$

Summing the above with respect to $i$ we obtain the formula (1.6). In case $Y$ is symmetric, the number of parameters in $Y_{i i}$ is

$$
\frac{1}{2} \sum_{j=1}^{n_{i}}\left[r_{i j}^{2}\left(2 e_{i j}-1\right)+r_{i j}+4 r_{i j} \sum_{k=j+1}^{n_{i}} r_{i k} e_{i k}\right] \text {. }
$$

Summing the above on $i$ we obtain (1.7).

Similarly, we can make use of Lemma 3 in proving (1.4) and (1.5).

We now prove

TheOREM 2. Let $A$ be as given in Theorem 1. Then the maximum number of linearly independent skew-symmetric matrices satisfying (1.8) or (1.11) is

$$
\frac{1}{2}(n-p)(n-p+1)
$$

Proof. In order to prove our result for $\operatorname{dim} \gamma\left(T^{2}\right)$, let $m_{i}=\sum_{j=1}^{n_{i}} \boldsymbol{r}_{i j} e_{i j}$ and consider 


$$
\begin{aligned}
m_{i}^{2}- & m_{i}-\sum_{j=1}^{n_{i}}\left[r_{i j}^{2}\left(2 e_{i j}-1\right)-r_{i j}+4 r_{i j} \sum_{k=j+1}^{n_{i}} r_{i k} e_{i k}\right] \\
= & \sum_{j=1}^{n_{i}}\left[r_{i j}^{2} e_{i j}^{2}+2 r_{i j} e_{i j} \sum_{k=j+1}^{n_{i}} r_{i k} e_{i k}-r_{i j} e_{i j}\right] \\
& -\sum_{j=1}^{n_{i}}\left[r_{i j}^{2}\left(2 e_{i j}-1\right)-r_{i j}+4 r_{i j} \sum_{k=j+1}^{n_{i}} r_{i k} e_{i k}\right] \\
= & \sum_{j=1}^{n_{i}}\left[r_{i j}^{2}\left(e_{i j}-1\right)^{2}-r_{i j}\left(e_{i j}-1\right)+2 r_{i j}\left(e_{i j}-2\right) \sum_{k=j+1}^{n_{i}} r_{i k} e_{i k}\right] .
\end{aligned}
$$

Now, it is clear that $r_{i j}^{2}\left(e_{i j}-1\right) \geqq r_{i j}\left(e_{i j}-1\right)$. The last term in the above expression will be negative only when $e_{i j}=1$. But we know that $e_{i 1}>e_{i 2}>\cdots>e_{i n_{i}}$, so that $e_{i j}$ will be 1 only for $j=n_{i}$. In that case $\sum_{k=j+1}^{n_{i}}$ does not appear, and we have

$$
\frac{1}{2} \sum_{j=1}^{n_{i}}\left[r_{i j}^{2}\left(2 e_{i j}-1\right)-r_{i j}+4 r_{i j} \sum_{k=j+1}^{n_{i}} r_{i k} e_{i k}\right] \leqq \frac{1}{2}\left(m_{i}^{2}-m_{i}\right) .
$$

This holds for $i=1, \cdots, p$.

To determine a bound on $\gamma(T)$, consider

$$
\begin{aligned}
& m_{i}^{2}-m_{i}-\sum_{j=1}^{n_{i}}\left[r_{i j}\left(r_{i j}-1\right) e_{i j}+2 r_{i j} \sum_{k=j+1}^{n_{i}} r_{i k} e_{i k}\right] \\
= & \sum_{j=1}^{n_{i}}\left[r_{i j}^{2} e_{i j}\left(e_{i j}-1\right)+2 r_{i j}\left(e_{i j}-1\right) \sum_{k=j+1}^{n_{i}} r_{i k} e_{i k}\right] \\
\geqq & 0, \text { since } e_{i j} \geqq 1 .
\end{aligned}
$$

Thus we have

$$
\frac{1}{2} \sum_{j=1}^{n_{i}}\left[r_{i j}\left(r_{i j}-1\right) e_{i j}+2 r_{i j} \sum_{k=j+1}^{n_{i}} r_{i k} e_{i k}\right] \leqq \frac{1}{2}\left(m_{i}^{2}-m_{i}\right)
$$

It may be observed that the upper bound is attained for $r_{i 1}=m_{i}, e_{i 1}=1$ and the remaining $e$ 's and $r$ 's all zero.

We have thus proved that

$$
\operatorname{dim} \gamma\left(T^{2}\right) \leqq \frac{1}{2} \sum_{i=1}^{p}\left(m_{i}^{2}-m_{i}\right)
$$

and

$$
\operatorname{dim} \gamma(T) \leqq \frac{1}{2} \sum_{i=1}^{p}\left(m_{i}^{2}-m_{i}\right)
$$

where $m_{i}$ is the multiplicity of the eigenvalue $\lambda_{i}$ of $A$.

Now we have to maximize $\sum_{i=1}^{p}\left(m_{i}^{2}-m_{i}\right)$ under the condition that 
$m_{1}+\cdots+m_{p}=n$, the order of $A$. Note that

$$
m_{i}^{2}-m_{i}=\left(m_{i}-1\right)^{2}+\left(m_{i}-1\right)
$$

and each $m_{i}-1 \geqq 0$. Hence, we have

$$
\sum_{i=1}^{p}\left(m_{i}-1\right)^{2} \leqq\left[\sum_{i=1}^{p}\left(m_{i}-1\right)\right]^{2}=(n-p)^{2} .
$$

Thus the maximum value of both $\operatorname{dim} \gamma\left(T^{2}\right)$ and $\operatorname{dim} \gamma(T)$ is

$$
\frac{1}{2}\left[(n-p)^{2}+(n-p)\right] \text {. }
$$

The bounds are achieved when $m_{1}=\cdots=m_{p-1}=1$ and $m_{p}=n-p+1$.

\section{REFERENCE}

1. O. Taussky and H. Zassenhaus, On the similarity transformation between a matrix and its transpose. Pacific J. Math. 9 (1959), 893-896.

The University of British Columbia, Vancouver, Canada AND

Muslim University, Aligarh, India 


\section{PACIFIC JOURNAL OF MATHEMATICS}

\section{EDITORS}

David GILbarg

Stanford University

Stanford, California

\section{F. H. BRowneLL}

University of Washington

Seattle 5, Washington

\section{A. L. Whiteman}

University of Southern California Los Angeles 7, California

\section{J. PAIGe}

University of California

Los Angeles 24, California

\section{ASSOCIATE EDITORS}
E. F. BECKENBACH
T. M. CHERRY
D. DERRY

\author{
E. HEWITT \\ A. HORN \\ L. NACHBIN
}

\author{
M. OHTSUKA \\ H. L. ROYDEN \\ M. M. SCHIFFER
}

E. SPANIER

E. G. STRAUS

F. WOLF

\section{SUPPORTING INSTITUTIONS}

\author{
UNIVERSITY OF BRITISH COLUMBIA \\ CALIFORNIA INSTITUTE OF TECHNOLOGY \\ UNIVERSITY OF CALIFORNIA \\ MONTANA STATE UNIVERSITY \\ UNIVERSITY OF NEVADA \\ NEW MEXICO STATE UNIVERSITY \\ OREGON STATE COLLEGE \\ UNIVERSITY OF OREGON \\ OSAKA UNIVERSITY \\ UNIVERSITY OF SOUTHERN CALIFORNIA
}

\author{
STANFORD UNIVERSITY \\ UNIVERSITY OF TOKYO \\ UNIVERSITY OF UTAH \\ WASHINGTON STATE COLLEGE \\ UNIVERSITY OF WASHINGTON \\ AMERICAN MATHEMATICAL SOCIETY \\ CALIFORNIA RESEARCH CORPORATION \\ HUGHES AIRCRAFT COMPANY \\ SPACE TECHNOLOGY LABORATORIES \\ NAVAL ORDNANCE TEST STATION
}

\footnotetext{
Mathematical papers intended for publication in the Pacific Journal of Mathematics should be typewritten (double spaced), and the author should keep a complete copy. Manuscripts may be sent to any one of the four editors. All other communications to the editors should be addressed to the managing editor, L. J. Paige at the University of California, Los Angeles 24, California.

50 reprints per author of each article are furnished free of charge; additional copies may be obtained at cost in multiples of 50 .
}

The Pacific Journal of Mathematics is published quarterly, in March, June, September, and December. The price per volume (4 numbers) is $\$ 12.00$; single issues, $\$ 3.50$. Back numbers are available. Special price to individual faculty members of supporting institutions and to individual members of the American Mathematical Society: $\$ 4.00$ per volume; single issues, $\$ 1.25$.

Subscriptions, orders for back numbers, and changes of address should be sent to Pacific Journal of Mathematics, 2120 Oxford Street, Berkeley 4, California.

Printed at Kokusai Bunken Insatsusha (International Academic Printing Co., Ltd.), No. 6, 2-chome, Fujimi-cho, Chiyoda-ku, Tokyo, Japan.

PUBLISHED BY PACIFIC JOURNAL OF MATHEMATICS, A NON-PROFIT CORPORATION

The Supporting Institutions listed above contribute to the cost of publication of this Journal, but they are not owners or publishers and have no responsibility for its content or policies. 


\section{Pacific Journal of Mathematics}

\section{Vol. 10, No. $4 \quad$ December, 1960}

M. Altman, An optimum cubically convergent iterative method of inverting a linear bounded operator in Hilbert space . . . . . . . . . . . . . . . . . . . . . . . . . . 1107

Nesmith Cornett Ankeny, Criterion for rth power residuacity ................. 1115

Julius Rubin Blum and David Lee Hanson, On invariant probability measures I . . . . . 1125

Frank Featherstone Bonsall, Positive operators compact in an auxiliary topology ..... 1131

Billy Joe Boyer, Summability of derived conjugate series . . . . . . . . . . . . . . . . 1139

Delmar L. Boyer, A note on a problem of Fuchs . . . . . . . . . . . . . . . . . 1147

Hans-Joachim Bremermann, The envelopes of holomorphy of tube domains in infinite

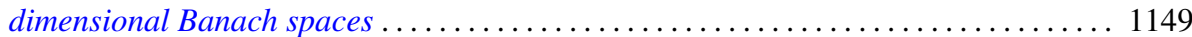

Andrew Michael Bruckner, Minimal superadditive extensions of superadditive

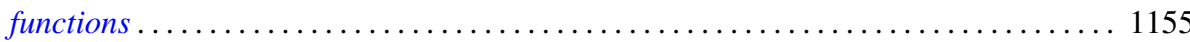

Billy Finney Bryant, On expansive homeomorphisms .................... 1163

Jean W. Butler, On complete and independent sets of operations in finite algebras . . . . . 1169

Lucien Le Cam, An approximation theorem for the Poisson binomial distribution ...... 1181

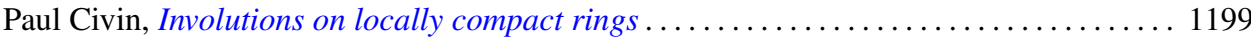

Earl A. Coddington, Normal extensions of formally normal operators . . . . . . . . . 1203

Jacob Feldman, Some classes of equivalent Gaussian processes on an interval ........ 1211

Shaul Foguel, Weak and strong convergence for Markov processes . . . . . . . . . . . 1221

Martin Fox, Some zero sum two-person games with moves in the unit interval ........ 1235

Robert Pertsch Gilbert, Singularities of three-dimensional harmonic functions . . . . . . . 1243

Branko Grünbaum, Partitions of mass-distributions and of convex bodies by

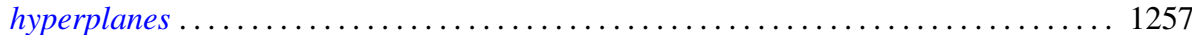

Sidney Morris Harmon, Regular covering surfaces of Riemann surfaces ........... 1263

Edwin Hewitt and Herbert S. Zuckerman, The multiplicative semigroup of integers

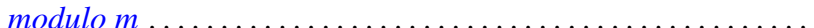

Paul Daniel Hill, Relation of a direct limit group to associated vector groups . ......... 1309

Calvin Virgil Holmes, Commutator groups of monomial groups . .

James Fredrik Jakobsen and W. R. Utz, The non-existence of expansive homeomorphisms

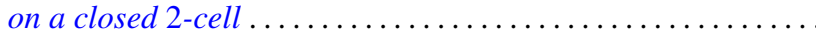

John William Jewett, Multiplication on classes of pseudo-analytic functions . . . . . . . 1323

Helmut Klingen, Analytic automorphisms of bounded symmetric complex domains . . . . 1327

Robert Jacob Koch, Ordered semigroups in partially ordered semigroups . . . . . . . . 1333

Marvin David Marcus and N. A. Khan, On a commutator result of Taussky and

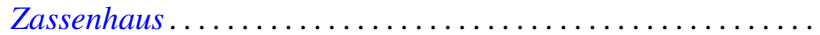

John Glen Marica and Steve Jerome Bryant, Unary algebras......

Edward Peter Merkes and W. T. Scott, On univalence of a continued fraction . . . . . . . 1361

Shu-Teh Chen Moy, Asymptotic properties of derivatives of stationary measures . . . . . 1371

John William Neuberger, Concerning boundary value problems . . . . . . . . . . . 1385

Edward C. Posner, Integral closure of differential rings . . . . . . . . . . . . . . . . . 1393

Marian Reichaw-Reichbach, Some theorems on mappings onto . . . . . . . . . . . . . 1397

Marvin Rosenblum and Harold Widom, Two extremal problems . . . . . . . . . . . . . . . . 1409

Morton Lincoln Slater and Herbert S. Wilf, A class of linear differential-difference

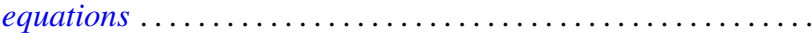

Charles Robson Storey, Jr., The structure of threads . . . . . . . . . . . . . . . . . . 1429

J. François Treves, An estimate for differential polynomials in $\partial / \partial z_{1},, \cdots, \partial / \partial z_{-} n \ldots \ldots 1447$

J. D. Weston, On the representation of operators by convolutions integrals . . . . . . . . 1453

James Victor Whittaker, Normal subgroups of some homeomorphism groups ......... 1469 